\section{Desigualdades sociais na distribuição espacial das hospitalizações por doenças respiratórias}

\author{
Social inequalities in spatial distribution of hospital \\ admissions due to respiratory diseases
}

\author{
Desigualdades sociales en la distribución espacial \\ de las hospitalizaciones por enfermedades \\ respiratorias
}

\begin{abstract}
To verify social inequalities in hospital admissions due to respiratory diseases in Salvador, Bahia State, Brazil, 2001-2007, an ecological study was conducted with information zones as the units of analysis. Information zones were stratified according to living conditions and analyzed by Poisson regression. Spatial distribution of hospitalization rates due to respiratory diseases ranged from 3.3 to 80.5/10,000. Asthma, pneumonia, and chronic obstructive pulmonary disease (COPD) showed heterogeneous spatial patterns, in which strata with the worst living conditions showed higher hospitalizations rates. The hospitalization rate for respiratory diseases was 2.4 times higher in zones with very low living conditions as compared to the wealthiest zone. There was a reduction in inequalities in hospital admissions for pneumonia and an increase for asthma and COPD. The sharp social gradient supports the hypothesis that socioeconomic factors are determinants of hospitalizations for respiratory diseases.
\end{abstract}

Respiratory Tract Diseases; Hospitalization; Residence Characteristics; Health Inequalities
Fernanda Pedro Antunes 1 Maria da Conceição Nascimento Costa ${ }^{1}$ Jairnilson Silva Paim 1 Ligia Maria Vieira-da-Silva 1 Álvaro Augusto Cruz 2 Márcio Natividade 1 Mauricio L. Barreto 1

\section{Resumo}

Para verificar as desigualdades sociais nas hospitalizações por doenças do aparelho respiratório em Salvador, Bahia, Brasil, 2001-2007, realizou-se estudo ecológico espacial, tendo como unidade de análise zonas de informação. Estas foram estratificadas considerando um indicador de condições de vida e analisadas por regressão de Poisson. A distribuição espacial das taxas de hospitalização por doenças do aparelho respiratório variou de 3,3 a 80,5/10 mil habitantes. Asma, pneumonia e doença pulmonar obstrutiva crônica (DPOC) apresentaram padrão espacial heterogêneo, no qual estratos de piores condições de vida apresentaram maior risco de ocorrência dessas internações. A taxa de hospitalização por doenças do aparelho respiratório foi 2,4 vezes maior no estrato de condições de vida muito baixas do que no primeiro estrato. Houve redução das desigualdades para pneumonia e aumento para asma e DPOC. A existência de forte gradiente social reforça a hipótese de que aspectos socioeconômicos são determinantes das hospitalizações por doenças do aparelho respiratório.

Doenças Respiratórias; Hospitalização; Distribuição Espacial da População; Desigualdades em Saúde 


\section{Introdução}

Estudos demonstram que áreas e grupos populacionais em situação socioeconômica desfavorável apresentam taxas de morbidade e mortalidade mais elevadas que aqueles em melhores condições de vida $1,2,3,4,5,6$, evidenciando a existência de desigualdades de perfis epidemiológicos entre diferentes regiões e grupos sociais.

Certamente que parte das desigualdades não deve ser vista como problema, pois expressa diferenças genéticas ou decorrentes das escolhas pessoais que sempre irão existir e não podem ser evitadas. As desigualdades que preocupam e devem ser alvo de políticas públicas são aquelas decorrentes da forma como a sociedade se organiza, já que, nesses casos, expressam não só a distribuição desigual e injusta das condições de vida e de trabalho 7 , como também o acesso diferenciado aos recursos que influenciam na qualidade de vida e saúde. Referem-se, desse modo, a diferenças desnecessárias, evitáveis e injustas, reconhecidas como iniquidades 8 .

As condições de vida dos diferentes grupos sociais podem ser expressas pelo espaço geográfico-social onde residem, uma vez que este representa uma instância da sociedade, contendo as várias dimensões da estrutura social, como a econômica, a política e a ideológica 9 . Assim sendo, alguns autores têm utilizado o espaço como uma categoria de análise no estudo das iniquidades em saúde 10,11,12.

Considerar a forma de organização social do espaço geográfico, estratificando os dados de saúde de acordo com essa categoria de análise, constitui uma das estratégias utilizadas para melhor compreensão da ocorrência e distribuição de agravos à saúde, permitindo desvendar, em parte, aspectos da dinâmica do processo saúde-doença que muitas vezes não podem ser evidenciados a partir dos dados agregados que não consideram variações espaciais 2 . $\mathrm{O}$ espaço geográfico é uma dimensão dinâmica que resulta de interações complexas entre fatores que definem a sua organização. Entendido como reflexo da sociedade global, constitui um determinante intrínseco das condições de vida da população 13,14. Desse modo, uma vez que os serviços de saúde são organizados em base espacial, o espaço geográfico pode permitir melhor adequação das ações de saúde às necessidades diferenciadas, auxiliando na adoção de intervenções mais equitativas referentes à atenção à saúde.

No que diz respeito às doenças do aparelho respiratório, há poucos estudos que utilizam essa abordagem metodológica, porém, em alguns, foi possível identificar áreas nas quais era maior o risco de internação ou a prevalência de algum tipo dessas doenças 15,16,17. Apesar de serem menos letais que as doenças cardiovasculares, as doenças respiratórias representaram a segunda causa de anos de vida perdidos por incapacidade no Brasil, ficando atrás apenas das doenças neuropsiquiátricas 18 , exercendo um grande peso sobre as condições de saúde e o sistema de saúde brasileiro. O cenário projetado por Mathers \& Loncar 19 evidencia, ainda, a importância desse grupo de causa no mundo nos próximos anos ao prever que as doenças pulmonares obstrutivas crônicas e as infecções respiratórias estarão entre as cinco principais causas de morte em 2030.

Desigualdades marcantes dos níveis de mortalidade por causas diversas, a exemplo das cardiovasculares e neoplasias, foram observadas entre as diferentes áreas da cidade de Salvador, capital do Estado da Bahia, Brasil 20,21, no entanto não foram encontradas investigações abordando as diferenças socioespaciais das doenças respiratórias neste município. Portanto, o presente estudo tem como objetivo identificar padrões na distribuição espacial das internações hospitalares pelo grupo das doenças do aparelho respiratório e principais doenças deste grupo (pneumonia, asma e doença pulmonar obstrutiva crônica - DPOC), em Salvador, nos anos de 2001 a 2007 -, além de verificar a existência de desigualdades socioespaciais nesta distribuição.

\section{Material e métodos}

Foi realizado um estudo de agregados espaciais, tendo como unidade de análise a zona de informação, que corresponde à divisão do território de Salvador em 93 áreas definidas pela Companhia de Desenvolvimento da Região Metropolitana (CONDER), com base em critérios geográficos, administrativos e de planejamento. Optou-se por essa unidade espacial em razão de ela apresentar correspondência com os setores censitários delimitados pelo Instituto Brasileiro de Geografia e Estatística (IBGE) e, principalmente, por possibilitar a comparação com os resultados de outras investigações sobre desigualdades sociais em saúde, realizadas anteriormente em Salvador. Foram analisadas as informações sobre doenças do aparelho respiratório (Classificação Internacional de Doenças, 10a Revisão - CID-10, capítulo X), pneumonia (J12-18), asma (J45-46) e DPOC (J43-44), referentes ao período 2001 a 2007 como um todo e, separadamente, para os anos 2001 e 2007. Este último foi o ano mais recente para o qual os dados foram disponibilizados quando da realização desta investigação. O estudo abrange toda a população residente nessa cidade, a terceira mais numerosa do país, com população 
estimada em aproximadamente 2.675.656 habitantes em 2010.

Com base em informações presentes nas Autorizações de Internações Hospitalares (AIH) de pacientes atendidos pelo Sistema Único de Saúde (SUS), fornecidas, em meio magnético (CD-ROM), pelo Departamento de Informação e Comunicação em Saúde da Secretaria de Saúde do Estado da Bahia (SESAB), foi possível georreferenciar endereços dos casos hospitalizados por doenças respiratórias, segundo zona de informação. Para tal, utilizou-se o programa Localiza (software desenvolvido pelo Instituto de Saúde Coletiva da Universidade Federal da Bahia, Salvador, Brasil), além do programa Google Earth (http://www.google.com/earth/index.html) para aqueles endereços cuja codificação não se deu automaticamente. Dados que não puderam ser georreferenciados, em virtude de não haver registro do endereço ou ser impossível sua identificação, foram descartados. representando 2,5\% do total de 66.332 casos.

A identificação de padrões na distribuição espacial das taxas de hospitalização por doenças do aparelho respiratório (por $10 \mathrm{mil} \mathrm{habi-}$ tantes) de cada zona de informação foi realizada mediante inspeção visual de mapas temáticos construídos com o emprego do software ArcView 3.3 (Environmental Systems Research Institute Inc., http://www.esri.com/software/arcview/). A existência de autocorrelação espacial dessas taxas foi avaliada pelo índice global de Moran, que varia de $-1 \mathrm{a}+1$, indicando se, quanto ao indicador estudado, áreas vizinhas apresentam maior semelhança do que o esperado em um padrão aleatório ${ }^{22}$. Em outras palavras, indica a presença de dependência espacial, entendida como a tendência que tem o valor de uma variável associada a uma determinada localização para assemelhar-se mais ao valor de suas amostras vizinhas, do que ao restante das localizações do conjunto amostral 23. Utilizou-se, para esta análise, o software S-Plus (Mathsoft Inc. Seattle, Estados Unidos), aceitando nível de 0,05 de significância.

A fim de verificar a existência de desigualdades sociais na distribuição espacial das internações por doenças do aparelho respiratório, construiu-se, para o ano 2000, um índice de condições de vida, indicador composto elaborado por Paim et al. 21 com base em cinco variáveis proxy de condições de vida: (1) renda (proporção de chefes de família em domicílios particulares permanentes, com rendimento médio mensal igual ou inferior a dois salários mínimos); (2) educação (proporção de pessoas de 10 a 14 anos alfabetizadas); (3) favelas (porcentagem de casas em aglomerados subnormal em relação ao total de domicílio); (4) saneamento (percentagem de domicílios com canalização interna ligada à rede global de abastecimento de água); (5) aglomeração domiciliar (razão entre o número médio de moradores por domicílio e o número médio de cômodos servindo de dormitório), por zona de informação. Entretanto, por não se dispor do número de cômodos servindo de dormitórios por setor censitário para o ano 2000, substituiu-se a razão "morador/quarto" por "morador/cômodo". Como só havia disponível o número de cômodos para o Município de Salvador como um todo, assumiu-se, para aquele ano, a mesma proporção de cômodos por domicílio em cada zona de informação observada em 1991.

Os valores dos indicadores favela, renda e morador/cômodo de cada zona de informação foram dispostos em ordem crescente, enquanto educação e saneamento foram organizados em ordem decrescente. Após essa etapa, receberam uma pontuação representada por valores consecutivos iniciados por 1 , de acordo com a posição assumida com esta ordenação e de modo que as melhores condições obtivessem a menor pontuação. A soma dessa pontuação resultou em um escore de índice de condições de vida para cada zona de informação; todos os escores foram, após dispostos em ordem crescente, agrupados em quartis de zona de informação relativamente homogênea, correspondentes aos estratos da população, classificados como melhores, intermediárias, baixas e muito baixas condições de vida (Figura 1).

As taxas de hospitalização de cada estrato de condições de vida foram padronizadas por idade, pelo método indireto, utilizando como padrão as taxas de hospitalização por faixa etária da população de Salvador do período estudado. A seguir, tomando como referência o estrato de melhores condições de vida, foram calculados os riscos relativos, utilizando-se análise de regressão de Poisson, por meio do programa Stata, versão 10 (Stata Corp., College Station, Estados Unidos), assumindo-se valor de $\mathrm{p}<0,05$. A população total de Salvador em 2001 e 2007 foi utilizada como denominador para o cálculo das taxas de hospitalização nos referidos anos (respectivamente, 2.436.390 e 2.738.827 habitantes).

O projeto deste estudo foi aprovado pelo Comitê de Ética em Pesquisa do Instituto de Saúde Coletiva, Universidade Federal da Bahia (ISC/ UFBA), sob o número 043-10/CEP-ISC.

\section{Resultados}

A taxa média de internação hospitalar por doenças do aparelho respiratório no Município de 

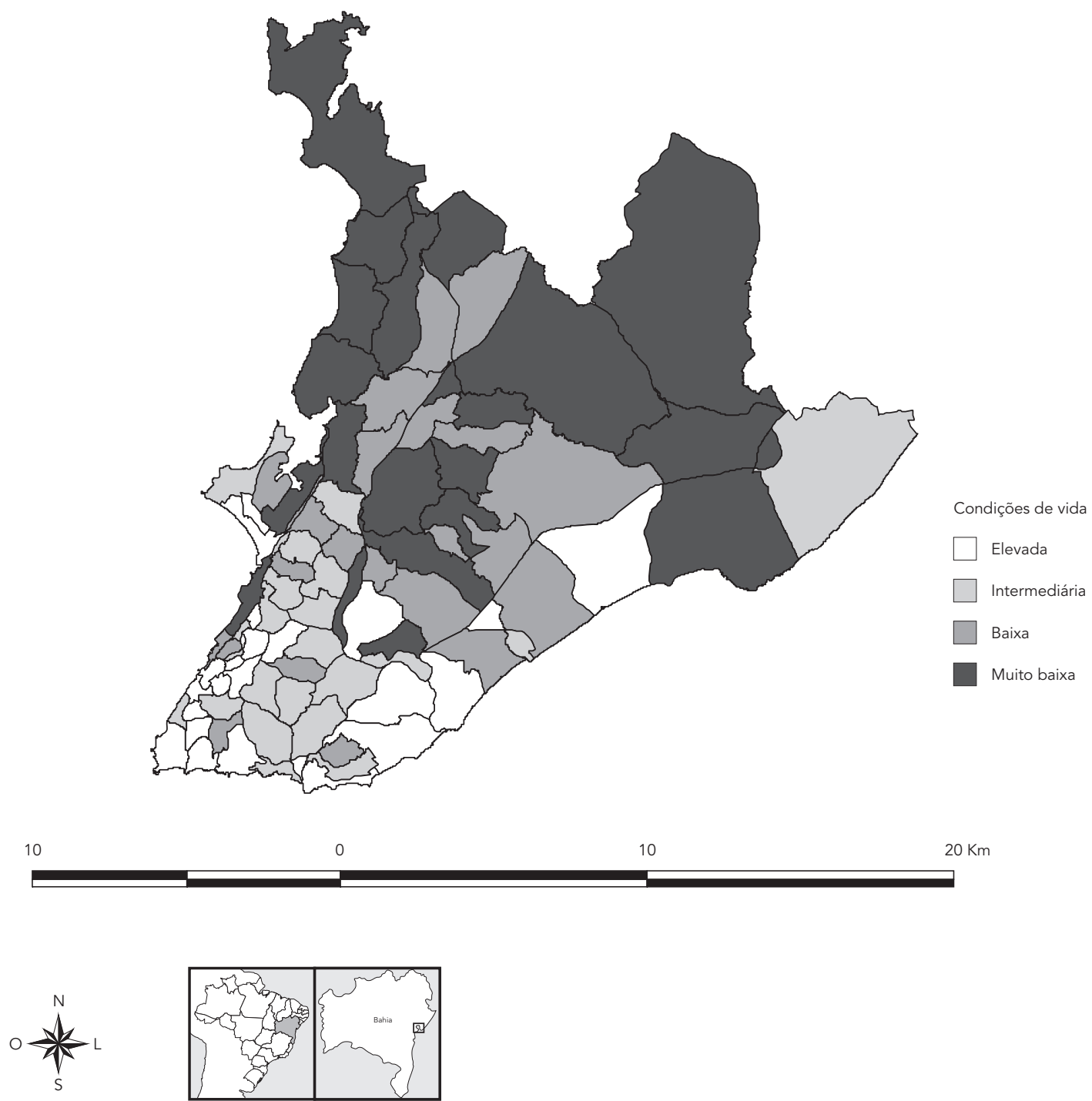

Salvador foi de 32,2/ 10 mil habitantes no período 2001-2007. Observa-se, porém, a existência de uma grande variabilidade na distribuição dessa taxa (3,3 a 80,5 internações por 10 mil habitantes) entre as zonas que compõem a capital baiana (Figura 2, Tabela 1). Verifica-se aumento das taxas de hospitalização por doenças do aparelho respiratório à medida que se avança do sul em direção ao norte da cidade. Assim, as zonas de informação com menor número de hospitalizações por doenças do aparelho respiratório concentram-se na orla marítima da cidade, com poucas exceções presentes no chamado "miolo", região situada na parte central de Salvador. Em contrapartida, as maiores taxas são vistas nas regiões voltadas para a Baía de Todos os Santos, que compreendem bairros como Comércio, Centro Histórico e Calçada, além de no subúrbio, região periférica de Salvador.

O índice de Moran, calculado para o período de 2001 a 2007, foi igual a 0,23 (valor de p < 0,001), indicando autocorrelação espacial positiva, ou seja, sinalizando que as taxas de hospitalização por doenças do aparelho respiratório das zonas 
Figura 2

Distribuição espacial das taxas de hospitalização por doenças respiratórias. Salvador, Bahia, Brasil, 2001 a 2007.

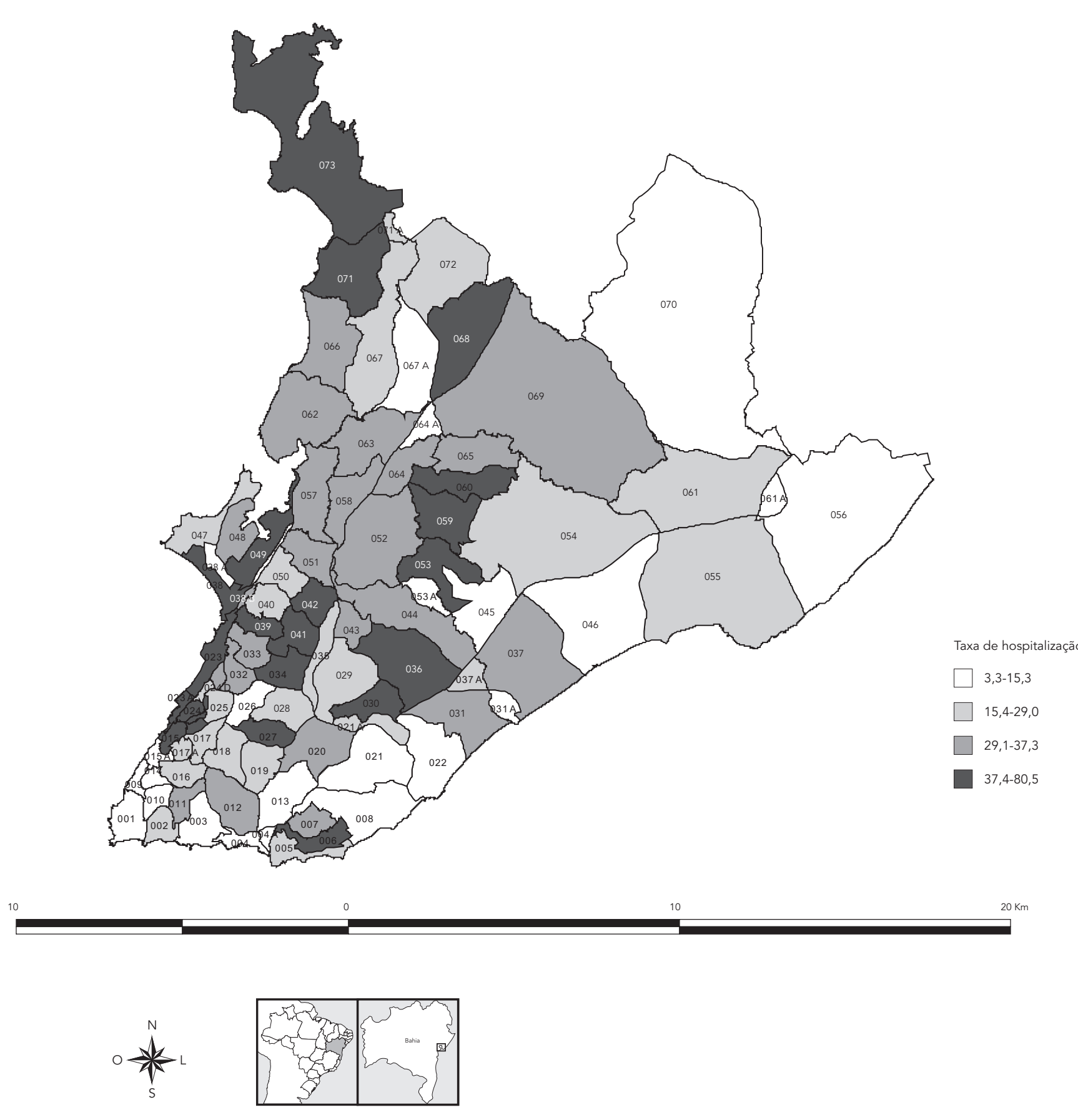

de informação não são independentes das taxas apresentadas nas regiões vizinhas. Isto foi verdadeiro mesmo quando as taxas foram padronizadas por idade $(0,19$; valor de $\mathrm{p}<0,002)$.
No que se refere à composição das áreas em estudo por quartil, observou-se que aquele que representa o estrato de condições de vida mais adequadas foi constituído por 24 zonas de 
Tabela 1

Lista das zonas de informação. Salvador, Bahia. Brasil.

\begin{tabular}{|c|c|c|c|c|c|}
\hline № & Nome & № & Nome & № & Nome \\
\hline 1 & Barra & $24 C$ & Baixa dos Sapateiros & 49 & Uruguai \\
\hline 2 & Apipema & $24 \mathrm{D}$ & Santo Antônio & 50 & São Caetano \\
\hline 3 & Ondina & $24 \mathrm{E}$ & Ladeira de Santana & 51 & Capelinha \\
\hline 4 & Vila Matos & 25 & Nazaré & 52 & Mata Escura \\
\hline $4 \mathrm{~A}$ & Parque Cruz Aguiar & 26 & Matatu & 53 & Sussuarana \\
\hline 5 & Amaralina & 27 & Cosme de Farias & $53 \mathrm{~A}$ & Estrada do Beiru \\
\hline 6 & Vale das Pedrinhas & 28 & Vila Laura & 54 & Canabrava \\
\hline 7 & Nordeste & 29 & Cabula & 55 & Piatã/Itapuã \\
\hline 8 & Pituba & 30 & Pernambués & 56 & Stella Maris \\
\hline 9 & Vitória & 31 & Boca do Rio & 57 & Lobato \\
\hline 10 & Graça & $31 \mathrm{~A}$ & Bolandeira & 58 & Campinas \\
\hline 11 & Federação & 32 & Barbalho & 59 & Pau da Lima \\
\hline 12 & $\begin{array}{l}\text { Engenho Velho da } \\
\text { Federação }\end{array}$ & 33 & Caixa D’Água & 60 & Sete de Abril \\
\hline 13 & Horto/Candeal & 34 & Quintas & 61 & Mussurunga \\
\hline 14 & Canela & 35 & Retiro & $61 \mathrm{~A}$ & Rótula do Aeroporto \\
\hline 15 & São Pedro & 36 & Narandiba & 62 & Plataforma \\
\hline $15 \mathrm{~A}$ & Forte de São Pedro & 37 & Pituaçu & 63 & Pirajá \\
\hline $15 B$ & Campo da Pólvora & $37 \mathrm{~A}$ & Imbuí & 64 & Dom Avelar \\
\hline 16 & Garcia & 38 & Calçada/Roma & $64 \mathrm{~A}$ & Porto Seco Pirajá \\
\hline 17 & Tororó & $38 \mathrm{~A}$ & Caminho de Areia & 65 & Castelo \\
\hline $17 \mathrm{~A}$ & Barris & $38 \mathrm{~B}$ & Baixa do Fiscal & 66 & Periperi \\
\hline 18 & Engenho Velho de Brotas & 39 & Pero Vaz & 67 & Parque São Bartolomeu \\
\hline 19 & Acupe de Brotas & 40 & Liberdade & $67 \mathrm{~A}$ & Represa do Cobre \\
\hline 20 & Campinas de Brotas & 41 & lapi & 68 & Valéria \\
\hline 21 & Itaigara & 42 & Fazenda Grande & 69 & Cajazeiras \\
\hline $21 \mathrm{~A}$ & Av. Tancredo Neves & 43 & São Gonçalo & 70 & Cia \\
\hline 22 & Stiep/Armação & 44 & $\begin{array}{c}\text { Tancredo Neves/ } \\
\text { Ergomadeira }\end{array}$ & 71 & Coutos \\
\hline 23 & Água de Meninos & 45 & $\begin{array}{c}\text { Centro Administrativo } \\
\text { da Bahia }\end{array}$ & 72 & Limite com a Usiba \\
\hline $23 \mathrm{~A}$ & Comércio & 46 & Patamares & 73 & Paripe \\
\hline 24. & Centro & 47 & Bonfim/Ribeira & & \\
\hline $24 \mathrm{~A}$ & Pelourinho & 48 & Massaranduba & & \\
\hline
\end{tabular}

informação, enquanto os demais, por 23 zonas de informação cada.

Houve diminuição das taxas de hospitalização por doenças do aparelho respiratório em todos os estratos de condições de vida, entre os anos de 2001 e 2007, de modo que a taxa média do município passou de 34,4 internações por 10 mil habitantes, em 2001, para 29,2, em 2007 (Tabela 2). A maior queda ocorreu no estrato de condições de vida intermediárias $(24,4 \%)$ e a menor, no estrato de condições de vida baixas (5,7\%).

A taxa de hospitalização por pneumonia no município estudado, por sua vez, passou de 14,8 internações por 10 mil habitantes para 15,6. O pequeno acréscimo observado nessa taxa $(5,4 \%)$ foi resultante do aumento ocorrido, principalmente, no primeiro e no terceiro quartis, com elevação de 13,2\% e 16,3\%, respectivamente.

As doenças respiratórias crônicas (asma e DPOC) tiveram diminuição de suas taxas de hospitalização. Todos os estratos apresentaram uma expressiva queda das hospitalizações por asma, superando uma variação de $60 \%$, sendo a mais acentuada a que ocorreu no estrato de melhor condição de vida (75\%), que passou de 2,8 para menos de uma internação por 10 mil habitantes. 
Tabela 2

Taxas * de hospitalização e suas variações por doenças do aparelho respiratório, asma, pneumonia e doença pulmonar obstrutiva crônica (DPOC), segundo condições de vida. Salvador, Bahia, Brasil, 2001 e 2007.

\begin{tabular}{|c|c|c|c|c|c|c|c|c|c|c|c|c|}
\hline \multirow[t]{2}{*}{ Estrato de condições de vida } & \multicolumn{3}{|c|}{ Doenças do aparelho respiratório } & \multicolumn{3}{|c|}{ Pneumonia } & \multicolumn{3}{|c|}{ Asma } & \multicolumn{3}{|c|}{ DPOC } \\
\hline & 2001 & 2007 & $\Delta \%$ & 2001 & 2007 & $\Delta \%$ & 2001 & 2007 & $\Delta \%$ & 2001 & 2007 & $\Delta \%$ \\
\hline Elevada & 16,7 & 14,8 & $-11,4$ & 5,3 & 6 & 13,2 & 2,8 & 0,7 & -75 & 1,7 & 0,7 & $-58,8$ \\
\hline Intermediária & 34,8 & 26,3 & $-24,4$ & 13,5 & 12,9 & $-4,4$ & 6 & 2,1 & -65 & 1,7 & 1,2 & $-29,4$ \\
\hline Baixa & 33,5 & 31,6 & $-5,7$ & 14,7 & 17,1 & 16,3 & 6,8 & 2,6 & $-61,8$ & 1,8 & 1,2 & $-33,3$ \\
\hline Muito baixa & 39,8 & 33,4 & -16 & 18,3 & 18,9 & 3,3 & 9,8 & 2,6 & $-73,5$ & 1,6 & 1,3 & $-18,8$ \\
\hline Salvador & 34,4 & 29,2 & $-15,1$ & 14,8 & 15,6 & 5,4 & 7,4 & 2,3 & $-68,9$ & 1,7 & 1,2 & $-29,4$ \\
\hline
\end{tabular}

Nota: taxas padronizadas por idade.

* Por 10 mil habitantes.

As taxas de hospitalização por DPOC também diminuíram em todos os estratos entre os anos 2001 e 2007. Novamente, o estrato de melhores condições de vida foi o responsável pela maior redução $(58,8 \%)$.

Nos dois anos referidos, observa-se que as taxas de hospitalização por doenças do aparelho respiratório e seus principais tipos foram mais elevados nos estratos de piores condições de vida. A existência desse gradiente socioeconômico permaneceu mesmo após a padronização das taxas por idade.

Na Tabela 3, observa-se a existência de gradiente social em todos os tipos de doenças do aparelho respiratório estudados, tanto em 2001 como em 2007. Em 2001, a taxa de hospitalização para esse grupo de doenças foi 2,4 vezes maior no estrato de condições de vida muito baixas, em relação ao de melhores condições de vida. Verificase, contudo, pequena redução do risco relativo no segundo e no quarto quartis, em 2007. Neste ano, o terceiro e o quarto estratos apresentaram os maiores riscos de hospitalização por pneumonia, sendo, respectivamente, 2,8 e 3,2 vezes maior do que no de condições de vida elevada. Entre os anos 2001 e 2007, houve redução do risco relativo nos estratos de condições de vida intermediárias e muito baixas. No caso da asma, em 2001, a taxa de hospitalização foi 3,6 vezes maior no quarto estrato e 2,4 vezes, no terceiro. $\mathrm{O}$ gradiente observado permaneceu, porém com crescimento, visto que a taxa de hospitalização por esta causa foi 3,6 vezes maior no estrato de baixas condições de vida, em 2007, ao passo que, no início do período, era apenas 2,4 vezes maior. Também houve aumento no segundo estrato, elevando-se de 2,2 para 2,8.

Ainda na Tabela 3, observa-se que não houve variação das taxas de hospitalização por DPOC nos diversos estratos de condições de vida, em 2001. Entretanto, em 2007, verificou-se um agravamento desse risco, sendo mais intenso naqueles de piores condições de vida.

\section{Discussão}

A heterogênea distribuição espacial das hospitalizações por doenças respiratórias e de seus principais tipos (asma, pneumonia e DPOC), em Salvador, mostrou-se associada às condições de vida das diferentes áreas deste município. Zonas de elevadas condições de vida concentraram baixas taxas de hospitalização, enquanto taxas mais elevadas foram observadas, principalmente, naquelas regiões onde as condições de vida eram baixas ou muito baixas. Padrão de distribuição espacial similar foi encontrado para outros indicadores de saúde, nesta capital, como a mortalidade infantil 24, mortalidade por doenças infecciosas e parasitárias 25 e por tuberculose 26 .

A demonstração da existência de autocorrelação espacial das taxas de hospitalização por doenças do aparelho respiratório corrobora a ideia de que áreas próximas tendem a apresentar taxas mais similares entre si, pois estas, diferentemente do que acontece entre regiões distantes, costumam compartilhar as mesmas características socioeconômicas e demográficas, favorecendo, assim, a formação de clusters ${ }^{23}$. Entende-se que estes não são apenas resultantes de escolhas pessoais em se aproximarem, mas constituem, principalmente, o produto de uma série de fatores sociais e políticos que favorecem a segregação espacial. O isolamento social e geográfico das áreas com piores condições de vida trazem importantes barreiras para o cuidado efetivo da saúde, como falhas de acesso aos serviços 
Tabela 3

Risco relativo e intervalo de $95 \%$ de confiança das hospitalizações por doenças do aparelho respiratório, pneumonia, asma e doença pulmonar obstrutiva crônica (DPOC), segundo condições de vida. Salvador, Bahia, Brasil, 2001 e 2007.

\begin{tabular}{|c|c|c|c|c|c|c|c|c|}
\hline \multirow[t]{2}{*}{ Estrato } & \multicolumn{2}{|c|}{ Doença do aparelho respiratório } & \multicolumn{2}{|c|}{ Pneumonia } & \multicolumn{2}{|c|}{ Asma } & \multicolumn{2}{|c|}{ DPOC } \\
\hline & 2001 & 2007 & 2001 & 2007 & 2001 & 2007 & 2001 & 2007 \\
\hline Elevada & - & - & - & - & - & - & - & - \\
\hline Intermediária & $2,1(1,9-2,3)$ & $1,8(1,6-2,0)$ & $2,5(2,1-3,0)$ & $2,1(1,8-2,5)$ & $2,2(1,7-2,7)$ & $2,8(1,8-4,4)$ & $1,0 *(0,7-1,4)$ & $1,8(1,1-2,8)$ \\
\hline Baixa & $2,0(1,8-2,2)$ & $2,1(1,9-2,3)$ & $2,8(2,3-3,3)$ & $2,8(2,4-3,3)$ & $2,4(1,9-3,1)$ & $3,6(2,4-5,6)$ & $1,0 *(0,7-1,4)$ & $1,9(1,2-2,9)$ \\
\hline Muito baixa & $2,4(2,2-2,6)$ & $2,3(2,1-2,5)$ & $3,4(2,9-4,0)$ & $3,2(2,7-3,6)$ & $3,6(2,8-4,4)$ & $3,6(2,4-5,5)$ & $1,0 *(0,7-1,3)$ & $2,0(1,3-3,2)$ \\
\hline
\end{tabular}

* Valor de $p<0,05$

básicos, a exemplo do transporte, que dificultam a busca por atenção à saúde 27 .

O aumento encontrado das admissões hospitalares por pneumonia pode, em parte, ser explicado pelo envelhecimento da população tendo em vista que os idosos constituem um grupo populacional mais susceptível às complicações desta doença 28 . Esse aumento também foi observado recentemente nos Estados Unidos 29, Espanha 30 e Inglaterra 31 , sendo mais marcante entre os idosos. Problemas de codificação das patologias, mudanças na organização dos serviços de saúde e, principalmente, o aumento da proporção de pessoas com comorbidades ${ }^{31}$, responsável pela maior vulnerabilidade dos indivíduos, também devem ser considerados.

No que se refere às desigualdades sociais nas hospitalizações por este grupo de causas, é possível que a redução observada para pneumonia seja um fato apenas aparentemente positivo, pois resultou do aumento mais acentuado de hospitalizações no estrato de melhores condições de vida, aproximando seus valores aos dos demais estratos. Ademais, é plausível supor que aquele estrato social também apresente melhor acesso aos serviços de saúde, o que pode ter influenciado no aumento das hospitalizações por esta causa. As maiores taxas, apesar disso, ainda concentram-se nas regiões de piores condições de vida, pois famílias com baixa renda não são capazes de providenciar bens, serviços e recursos suficientes, como alimentação adequada e menor aglomeração intradomiciliar, que contribuiriam para a prevenção de eventos adversos à saúde.

Diferentemente da pneumonia, as doenças respiratórias crônicas apresentaram queda das taxas de hospitalização em todos os estratos, sendo superior a $75 \%$ e $58 \%$ no estrato melhores condições de vida, para asma e DPOC, respectivamente. A ampliação dos conhecimentos acer- ca do manejo dessas doenças, juntamente com a introdução de novos medicamentos no mercado para seu controle, pode ter colaborado para a diminuição dessas internações, principalmente no primeiro estrato, que, possivelmente, conta com maior acesso aos serviços e tratamentos disponíveis. As maiores taxas encontradas nos estratos baixas e muito baixas condições de vida devem-se, dentre outros fatores, a dificuldades no acesso ao tratamento oportuno, condições precárias de moradia, baixa escolaridade, a qual dificulta a percepção dos sintomas antes de seu agravamento, baixa renda, impedindo o controle dessas doenças com os medicamentos inalatórios disponíveis, e maior proporção de tabagismo. Apesar da redução de uma forma geral do hábito de fumar no Brasil, este ainda se encontra mais concentrado entre os grupos com menor escolaridade, que podem ser também os mais pobres 32 .

Em relação à DPOC, alguns autores chamam atenção para o sub-registro dessa doença entre pacientes hospitalizados, principalmente, aqueles admitidos com alguma condição respiratória aguda 33,34. Esta pode ser uma explicação plausível para Salvador, onde o aumento das hospitalizações por pneumonia pode estar levando à confusão de diagnósticos e sub-registro da DPOC. A adoção de um programa de intervenção em saúde pública para o controle da asma grave no município ${ }^{35}$, em 2003, tem sido referida como capaz de reduzir as hospitalizações por esta enfermidade nos estratos onde reside a população mais pobre.

Apesar disso, as taxas de admissão por asma continuam a apresentar forte gradiente social, sendo mais elevadas tanto em 2001, quanto em 2007, tendo, inclusive, acentuado-se neste último ano. O mesmo comportamento foi observado para a DPOC, em 2007. Outros estudos também demonstraram a existência de iniquidades 
nas internações por asma em diferentes localidades 27,36 , sendo maior do que as disparidades encontradas na sua prevalência, sugerindo que, uma vez estabelecida, muitos fatores convergem para o aumento da gravidade nas populações mais carentes 36 . Nível educacional baixo, barreiras linguísticas entre pacientes e médicos e falhas na percepção dos sintomas pelos mais carentes são alguns dos fatores que interferem na habilidade de controle da doença pelo próprio indivíduo e na comunicação médico-paciente 27 . $O$ alto custo dos medicamentos para controle da asma grave e diferenças do ambiente intradomiciliar, como maior umidade, poeira e aglomeração, podem explicar, também, as maiores taxas encontradas nas áreas em desvantagem social.

Salienta-se a necessidade de cautela quando da interpretação dos resultados deste estudo, em razão de possíveis falhas de georreferenciamento dos endereços dos pacientes decorrentes de preenchimento incorreto ou insuficiente dos registros hospitalares. Outro problema metodológico refere-se à utilização, como unidade espacial, de zona de informação, que são zonas geográficoadministrativas para fins de orientação do planejamento; apesar de apresentarem características socioeconômicas internas próximas, elas não são homogêneas. Deve, ainda, ser considerada a possibilidade de problemas como erros de diagnóstico ou de codificação e duplicidade de casos, dentre outros. Em adição, destaca-se o fato de que os dados analisados no presente estudo referem-se apenas às hospitalizações do sistema público de saúde brasileiro, o SUS, cuja cobertura é superior a 60\% (Departamento de Informática do SUS. Sistema de Informações Hospitalares. http://www.datasus.gov.br, acessado em 15/ Mai/2010). Ao se tratar exclusivamente de hospitalizações do SUS, este estudo não engloba a parcela da população de Salvador que possui plano de saúde ou que pode pagar pelos serviços hospitalares, tendo, portanto, melhor nível socioeconômico. Dessa forma, as desigualdades apontadas podem estar superestimadas, visto que, possivelmente, as regiões de melhores condições de vida não tiveram as possíveis hospitalizações por doenças do aparelho respiratório analisadas. Entretanto, foi possível identificar a existência de um gradiente social, mesmo após a padronização das taxas pela idade, o que reforça a ideia de que fatores socioeconômicos, mediados pelas condições de vida, são determinantes das admissões hospitalares por doenças respiratórias. Ressalva-se, ainda, que os resultados aqui apresentados não devem ser inferidos para indivíduos, pois o fato de eles pertencerem à mesma região ou estrato não os põe em igual situação de risco.

Apesar do declínio das internações por doenças do aparelho respiratório observado em Salvador, a manutenção das desigualdades entre os estratos de condições de vida e, no caso da asma e DPOC, seu agravamento trazem à tona a ineficiência das intervenções públicas até então adotadas para a redução das iniquidades. As doenças respiratórias, em especial, as crônicas, têm mecanismos causais complexos; assim, para que sejam captadas mudanças nos padrões de distribuição espacial das suas internações, é necessário que as ações de saúde envolvam diferentes áreas, como ambiente domiciliar e aspectos da comunidade. 


\section{Resumen}

Con el fin de verificar las desigualdades sociales en las hospitalizaciones por enfermedades del aparato respiratorio en Salvador, Bahía, Brasil, durante 2001-2007, se realizó un estudio ecológico espacial, teniendo como unidad de análisis zonas de información. Estas fueron estratificadas considerando un indicador de condiciones de vida y analizadas por la regresión de Poisson. La distribución espacial de las tasas de hospitalización por enfermedades del aparato respiratorio varió de 3,3/10.000 a 80,5/10.000 habitantes. Asma, neumonía y enfermedad pulmonar obstructiva crónica (EPOC) presentaron un padrón espacial heterogéneo, en el que los estratos de peores condiciones de vida presentaron un mayor riesgo de ocurrencia en este tipo de internamientos. La tasa de hospitalización por enfermedades del aparato respiratorio fue un 2,4 veces mayor en el estrato con condiciones de vida muy bajas que en el primer estrato. Hubo una reducción de las desigualdades en el caso de la neumonía y un aumento en el asma y EPOC. La existencia de un fuerte gradiente social refuerza la hipótesis de que los aspectos socioeconómicos son determinantes en las hospitalizaciones por enfermedades del aparato respiratorio.

Enfermedades Respiratorias; Hospitalización;

Distribución Espacial de la Población;

Desigualdades en la Salud

\section{Referências}

1. Marmot MG, Rose G, Shipley M, Hamilton PJS. Employment grade and coronary heart disease in British civil servants. J Epidemiol Community Health 1978; 32:244-9.

2. Núñez N. Perfiles diferenciales de mortalidad según condiciones de vida. Venezuela 1983 y 1990. Gac Méd Caracas 1997; 105:189-201.

3. Carneiro de Oliveira D, Salgado Barreira A, Taracido Trunk M, Figueiras Guzmán A. Impact of socioeconomic inequities on the mortality of the City of Fortaleza, Ceara, Brazil during 2007. Rev Esp Salud Pública 2010; 84:443-52.

\section{Colaboradores}

F. P. Antunes, M. C. N. Costa, J. S. Paim, L. M. Vieira-daSilva, A. A. Cruz e M. L. Barreto contribuíram na concepção e execução do projeto; análise e interpretação dos dados; redação do artigo; revisão crítica relevante do conteúdo intelectual e aprovação da versão final. M. Natividade colaborou na análise e interpretação dos dados e aprovação da versão final.
4. Choi MH, Cheong KS, Cho BM, Hwang IK, Kim $\mathrm{CH}$, Kim $\mathrm{MH}$, et al. Deprivation and mortality at the town level in Busan, Korea: an ecological study. J Prev Med Public Health 2011; 44:242-8.

5. Weightman AL, Morgan HE, Shepherd MA, Kitcher H, Roberts C, Dunstam FD. Social inequality and infant health in the UK: systematic review and meta-analyses. BMJ Open 2012; 2:e000964.

6. McCartney D, Scarborough P, Webster P, Rayner M. Trends in social inequalities for premature coronary heart disease mortality in Great Britain, 19942008: a time trend ecological study. BMJ Open 2012; 2:e00737. 
7. Possas C. Epidemiologia e sociedade: heterogeneidade estrutural e saúde no Brasil. São Paulo: Editora Hucitec; 1989.

8. Whitehead M. The concepts and principles of equity and health. Copenhagen: World Health Organization Regional Office for Europe; 2000.

9. Paim J. Abordagens teórico-conceituais em estudos de condições de vida e saúde: notas para reflexão e ação. In: Barata RB, organizador. Condições de vida e situação de Saúde. Rio de Janeiro: ABRASCO; 1997. (Saúde Movimento, 4).

10. Butler DC, Petterson S, Phillips RL, Bazemore AW. Measures of social deprivation that predict health care access and need within a rational area of primary care service delivery. Health Serv Res 2012; [Epub ahead of print].

11. Kirkbride JB, Jones PB, Ullrich S, Coid JW. Social deprivation, inequality, and the neighborhood-level incidence of psychotic syndromes in East London. Schizophr Bull 2012; [Epub ahead of print].

12. Queiroga RPF, Sá LD, Nogueira JA, Lima ERV, Silva ACO, Pinheiro PGOD, et al. Distribuição espacia da tuberculose e a relação com condições de vida na área urbana do município de Campina Grande - 2004 a 2007. Rev Bras Epidemiol 2012; 15:222-32.

13. Santos M. Espaço e método. 3a Ed. São Paulo: Nobel; 1985.

14. Santos M. A natureza do espaço - técnica e tempo. Razão e emoção. São Paulo: Editora Hucitec; 1996.

15. Mukai AO, Alves KSC, Nascimento LFC. Análise espacial das internações por pneumonia no Vale da Paraíba (SP). J Bras Pneumol 2009; 35:753-8.

16. Crighton EJ, Elliott SJ, Moineddin R, Kanaroglou P, Upshur REG. An exploratory spatial analysis of pneumonia and influenza hospitalizations in Ontario by age and gender. Epidemiol Infect 2007 135:253-61.

17. Disano J, Goulet J, Muhajarine N, Neudorf C, Harvey J. Social-economic status and rates of hospita admission for chronic disease in urban Canada. Can Nurse 2010; 106:24-9.

18. Schramm JMA, Oliveira AF, Leite IC, Valente JG, Gadelha AMJ, Portela MC, et al. Transição epidemiológica e o estudo da carga de doenças no Brasil. Ciênc Saúde Coletiva 2004; 9:897-908.

19. Mathers CD, Loncar D. Projections of global mortality and burden of disease from 2002 to 2030 . PLoS Med 2006; 3:e442.

20. Costa MCN, Azi PA, Paim J, Vieira-da-Silva LM. Mortalidade infantil e condições de vida: a reprodução das desigualdades sociais em saúde na década de 90. Cad Saúde Pública 2001; 17:555-67.

21. Paim J, Vieira-da-Silva LM, Costa MCN, Prata PR, Lessa I. Desigualdades na situação de saúde do município de Salvador e suas relações com as condições de vida. Rev Ciênc Med Biol 2003; 2:30-9.

22. Cliff AD, Ord JK. Spatial process: models and applications. London: Pion; [1981?].

23. Câmara G, Monteiro AM, Fucks SD, Carvalho MS Análise espacial e geoprocessamento. In: Druck S, Câmara G, Monteiro AM, Carvalho MS, organizadores. Análise espacial de dados geográficos. http://www.dpi.inpe.br/gilberto/livro/analise/ acessado em 20/Jul/2010).
24. Gonçalves AC, Costa MCN, Braga JU. Análise da distribuição espacial da mortalidade neonatal e de fatores associados, em Salvador, Bahia, Brasil, no período 2000-2006. Cad Saúde Pública 2011; 27:1581-92

25. Teixeira MG, Meyer MA, Costa MCN, Paim JS, Vieira-da-Silva LM. Mortalidade por doenças infecciosas e parasitárias em Salvador - Bahia: evolução e diferenciais intra-urbanos segundo condições de vida. Rev Soc Bras Med Trop 2002; 35:491-7.

26. Mota FF, Vieira-da-Silva LM, Paim JS, Costa MCN. Distribuição espacial da mortalidade por tubercu lose em Salvador, Bahia, Brasil. Cad Saúde Pública 2003; 19:915-22.

27. Bryant-Stephens T. Asthma disparities in urban environments. J Allergy Clin Immunol 2009; 123 1199-206.

28. Solh AE, Pineda L, Bouquin P, Mankowski C. Determinants of short and long term functional recovery after hospitalization for community-acquired pneumonia in the elderly: role of inflammatory markers. BMC Geriatrics 2006; 6:12.

29. Fry AM, Shay DK, Holman RC, Curns AT, Anderson LJ. Trends for hospitalizations for pneumonia among persons aged 65 years or older in the United States, 1988-2002. JAMA 2005; 294:2712-9.

30. Ochoa-Gondar O, Vila-Córcoles A, de Diego C, Arija V, Maxenchs M, Grive M, et al. The burden of community-acquired pneumonia in the elderly: the Spanish EVAN-65 Study. BMC Public Health 2008; 8:222.

31. Trotter CL, Stuart JM, George R, Miller E. Increasing hospital admissions for pneumonia, England. Emerg Infect Dis 2008; 14:727-33.

32. Iglesias R, Jha P, Pinto M, Costa e Silva VL, Godinho, J. Controle do tabagismo no Brasil. Washington DC: Rede de Desenvolvimento Humano/Banco Mundial; 2007.

33. Thomsen RW, Lange P, Hellquist B, Frausing E, Bartels PD, Krog BR, et al. Validity and underrecording of diagnosis of COPD in the Danish National Patient Registry. Respir Med 2011; 105:1063-8.

34. Bednarek M, Maciejewski J, Wozniak M, Kuca P, Zielinski J. Prevalence, severity and underdiagnosis of COPD in the primary care setting. Thorax 2008; 63:402

35. Cruz AA, Souza-Machado A, Franco R, SouzaMachado C, Ponte EV, Moura Santos P, et al. The impact of a program for control of asthma in a low-income setting. World Allergy Organ J 2010; 3:167-74.

36. Gold DR, Wright R. Population disparities in asthma. Annu Rev Public Health 2005; 26:89-113.

Recebido em 22/Ago/2012

Versão final reapresentada em 06/Jan/2013

Aprovado em 28/Jan/2013 